\title{
ROOT SUPERMULTIPLICITIES AND CORRESPONDING COMBINATORIAL IDENTITIES FOR SOME BORCHERDS SUPERALGEBRAS
}

\author{
Neelacanta Sthanumoorthy and Kandasamy Priyadharsini \\ University of Madras, Chennai, India
}

\begin{abstract}
In this paper, root supermultiplicities and corresponding combinatorial identities for the Borcherds superalgebras which are extensions of $A_{2}$ and $A_{3}$ are found out. Moreover, superdimension formula for a Borcherds superalgebra which is an extension of a particular hyperbolic Kac-Moody algebra is also computed.
\end{abstract}

\section{INTRODUCTION}

In 1977, theory of Lie superalgebras was constructed by Kac. The theory of Lie superalgebras can also be seen in Scheunert ([24]) in a detailed manner. The notion of Kac-Moody superalgebras was introduced by Kac ([7]) and therein the Weyl-Kac character formula for the irreducible highest weight modules with dominant integral highest weight which yields a denominator identity when applied to 1-dimensional representation was also derived. Borcherds $([1,2])$ proved a character formula called Weyl-Borcherds formula which yields a denominator identity for a generalized Kac-Moody algebra. Miyamoto ([23]) introduced the theory of generalized Lie superalgebra version of the generalized Kac-Moody algebras (Borcherds algebras) and had shown that the transformed Borcherds superalgebras and Borcherds superalgebras have many similar properties. A homological theory for the graded Lie algebras was developed by Kang ([10]) and a closed form root multiplicity formula for all symmetrizable generalized Kac-Moody algebras was derived in Kang ([12]). Kim and Shin ([21]) derived a recursive dimension formula for all graded Lie algebras. Kang and Kim ([18]) calculated the dimension

2010 Mathematics Subject Classification. 17B65, $17 \mathrm{~B} 67$.

Key words and phrases. Borcherds superalgebras, colored superalgebras, root supermultiplicities. 
formula for graded Lie algebras. Computation of root multiplicities of many Kac-Moody algebras and generalized Kac-Moody algebras can be seen in Kass et al. ([20]), Kang ([11,13-15]), S. J. Kang and D. J. Melville ([19]), Frenkel and Kac ([4]), Feingold and Frenkel ([3]), Kac and Wakimoto ([9]), Hontz and Misra ([5]), Sthanumoorthy and Uma Maheswari ([26]), Sthanumoorthy et al. $([31,32])$ and Sthanumoorthy and Lilly $([34])$. Computation of root multiplicities of Borcherds superalgebras was found in Sthanumoorthy et al. ([36]). Some properties of different classes of root systems and their classifications for Kac-Moody algebras and Borcherds Kac-Moody algebras were studied in Sthanumoorthy and Uma Maheswari ([25]) and Sthanumoothy and Lilly $([27-30,33])$. Also, properties of different root systems and complete classifications of special, strictly and purely imaginary roots of Borcherds KacMoody Lie superalgebras which are extensions of Kac-Moody Lie algebras were explained in Sthanumoorthy et al. ([35,37]) and Sthanumoorthy and Priyadharsini ([38, 39]). Moreover, Kang ([16]) obtained a superdimension formula for the homogeneous subspaces of the graded Lie superalgebras, which enabled one to study the structure of the graded Lie superalgebras in a unified way. Using the Weyl-Kac-Borcherds formula and the denominator identity for the Borcherds superalgerbas, Kang and Kim ([17]) derived a dimension formula and combinatorial identities for the Borcherds superalgebras and found out the root multiplicities for Monstrous Lie superalgebras.

In this paper, we compute dimensional formulae, root supermultiplicities and corresponding combinatorial identities for the Borcherds superalgebras which are extensions of Kac-Moody algebras $A_{2}, A_{3}$. Moreover, we compute a dimension formula for a Borcherds superalgebra which is extension of hyperbolic Kac-Moody algebra. Particular cases of these Borcherds superalgebras were considered in Sthanumoorthy et al. ([36]) and therein only dimension formulas were found out. Throughout this paper we use mainly the results from Kang and Kim ([17]).

\section{PRELIMINARIES}

In this section, we give some basic concepts of Borcherds superalgebras as in Kang and Kim ([17]).

Definition 2.1. Let I be a countable (possibly infinite) index set. A real square matrix $A=\left(a_{i j}\right)_{i, j \in I}$ is called Borcherds-Cartan matrix if it satisfies:

(1) $a_{i i}=2$ or $a_{i i} \leq 0$ for all $i \in I$,

(2) $a_{i j} \leq 0$ if $i \neq j$ and $a_{i j} \in \mathbb{Z}$ if $a_{i i}=2$,

(3) $a_{i j}=0 \Leftrightarrow a_{j i}=0$.

We say that an index $i$ is real if $a_{i i}=2$ and imaginary if $a_{i i} \leq 0$. We denote by

$$
I^{\mathrm{re}}=\left\{i \in I \mid a_{i i}=2\right\}, I^{\mathrm{im}}=\left\{i \in I \mid a_{i i} \leq 0\right\} .
$$


Let $\underline{m}=\left\{m_{i} \in \mathbb{Z}_{>0} \mid i \in I\right\}$ be a collection of positive integer such that $m_{i}=1$ for all $i \in I^{\mathrm{re}}$. We call $\underline{m}$, charge of $A$.

Definition 2.2. A Borcherds-Cartan matrix $A$ is said to be symmetrizable if there exists a diagonal matrix $D=\operatorname{diag}\left(\epsilon_{i} ; i \in I\right)$ with $\epsilon_{i}>0(i \in I)$ such that $D A$ is symmetric.

Let $C=\left(c_{i j}\right)_{i, j \in I}$ be a complex matrix satisfying $c_{i j} c_{j i}=1$ for all $i, j \in I$. Therefore, we have $c_{i i}= \pm 1$ for all $i \in I$. We call $i \in I$ an even index if $c_{i i}=1$ and an odd index if $c_{i i}=-1$.

We denote by $I^{\text {even }}\left(I^{\text {odd }}\right)$ the set of all even (odd) indices.

Definition 2.3. A Borcherds-Cartan matrix $A=\left(a_{i j}\right)_{i, j \in I}$ is restricted (or colored) with respect to $C$ if it satisfies:

If $a_{i i}=2$ and $c_{i i}=-1$ then $a_{i j}$ are even integers for all $j \in I$. In this case, the matrix $C$ is called a coloring matrix of $A$.

Let $\mathfrak{h}=\left(\oplus_{i \in I} \mathbb{C} h_{i}\right) \oplus\left(\oplus_{i \in I} \mathbb{C} d_{i}\right)$ be a complex vector space with a basis $\left\{h_{i}, d_{i} ; i \in I\right\}$, and for each $i \in I$, define a linear functional $\alpha_{i} \in \mathfrak{h}^{\star}$ by

$$
\alpha_{i}\left(h_{j}\right)=a_{j i}, \alpha_{i}\left(d_{j}\right)=\delta_{i j} \text { for all } j \in I .
$$

If $A$ is symmetrizable, then there exists a symmetric bilinear form (.|.) on $\mathfrak{h}^{\star}$ satisfying $\left(\alpha_{i} \mid \alpha_{j}\right)=\epsilon_{i} a_{i j}=\epsilon_{j} a_{j i}$ for all $i, j \in I$.

Definition 2.4. Let $Q=\oplus_{i \in I} \mathbb{Z} \alpha_{i}$ and $Q_{+}=\sum_{i \in I} \mathbb{Z}_{\geq 0} \alpha_{i}, Q_{-}=-Q_{+}$. $Q$ is called the root lattice.

The root lattice $Q$ becomes a (partially) ordered set by putting $\lambda \geq \mu$ if and only if $\lambda-\mu \in Q_{+}$.

The coloring matrix $C=\left(c_{i j}\right)_{i, j \in I}$ defines a bimultiplicative form $\theta$ : $Q \times Q \rightarrow C^{X}$ by

$$
\begin{aligned}
\theta\left(\alpha_{i}, \alpha_{j}\right) & =c_{i j} \text { for all } i, j \in I, \\
\theta(\alpha+\beta, \gamma) & =\theta(\alpha, \gamma) \theta(\beta, \gamma), \\
\theta(\alpha, \beta+\gamma) & =\theta(\alpha, \beta) \theta(\alpha, \gamma)
\end{aligned}
$$

for all $\alpha, \beta, \gamma \in Q$. Note that $\theta$ satisfies

$$
\theta(\alpha, \beta) \theta(\beta, \alpha)=1 \text { for all } \alpha, \beta \in Q,
$$

since $c_{i j} c_{j i}=1$ for all $i, j \in I$.

In particular $\theta(\alpha, \alpha)= \pm 1$ for all $\alpha \in Q$.

We say $\alpha \in Q$ is even if $\theta(\alpha, \alpha)=1$ and odd if $\theta(\alpha, \alpha)=-1$.

DeFinition 2.5. A $\theta$-colored Lie superalgebra is a $Q$-graded vector space $L=\oplus_{\alpha \in Q} L_{\alpha}$ together with a bilinear product $[\cdot, \cdot]: L \times L \rightarrow L$ satisfying

$$
\begin{aligned}
{\left[L_{\alpha}, L_{\beta}\right] } & \subset L_{\alpha+\beta}, \\
{[x, y] } & =-\theta(\alpha, \beta)[y, x], \\
{[x,[y, z]] } & =[[x, y], z]+\theta(\alpha, \beta)[y,[x, z]]
\end{aligned}
$$


for all $\alpha, \beta \in Q$ and $x \in L_{\alpha}, y \in L_{\beta}, z \in L$.

In a $\theta$-colored Lie superalgebra $L=\oplus_{\alpha \in Q} L_{\alpha}$, for $x \in L_{\alpha}$, we have $[x, x]=$ 0 if $\alpha$ is even and $[x,[x, x]]=0$ if $\alpha$ is odd.

DeFinition 2.6. The universal enveloping algebra $U(L)$ of a $\theta$-colored Lie superalgebra $L$ is defined to be $T(L) / J$, where $T(L)$ is the tensor algebra of $L$ and $J$ is the ideal of $T(L)$ generated by the elements $[x, y]-x \otimes y+\theta(\alpha, \beta) y \otimes x$ $\left(x \in L_{\alpha}, y \in L_{\beta}\right)$.

Definition 2.7. The Borcherds superalgebra $\mathfrak{g}=\mathfrak{g}(A, \underline{m}, C)$ associated with the symmetrizable Borcherds-Cartan matrix $A$ of charge $\underline{m}=\left(m_{i} ; i \in\right.$ $I)$ and the coloring matrix $C=\left(c_{i j}\right)_{i, j \in I}$ is the $\theta$-colored Lie superalgebra generated by the elements $h_{i}, d_{i}(i \in I), e_{i k}, f_{i k}\left(i \in I, k=1,2, \cdots, m_{i}\right)$ with defining relations:

$$
\begin{array}{ll}
{\left[h_{i}, h_{j}\right]} & =\left[h_{i}, d_{j}\right]=\left[d_{i}, d_{j}\right]=0, \\
{\left[h_{i}, e_{j l}\right]} & =a_{i j} e_{j l},\left[h_{i}, f_{j l}\right]=-a_{i j} f_{j l}, \\
{\left[d_{i}, e_{j l}\right]} & =\delta_{i j} e_{j l},\left[d_{i}, f_{j l}\right]=-\delta_{i j} f_{j l}, \\
{\left[e_{i k}, f_{j l}\right]} & =\delta_{i j} \delta_{k l} h_{i} \\
\left(a d e_{i k}\right)^{1-a_{i j}} e_{j l} & =\left(a d f_{i k}\right)^{1-a_{i j}} f_{j l}=0 \text { if } a_{i i}=2 \text { and } i \neq j, \\
{\left[e_{i k}, e_{j l}\right]} & =\left[f_{i k}, f_{j l}\right]=0 \text { if } a_{i j}=0
\end{array}
$$

for $i, j \in I, k=1, \cdots, m_{i}, l=1, \cdots, m_{j}$.

The abelian subalgebra $\mathfrak{h}=\left(\oplus_{i \in I} \mathbb{C} h_{i}\right) \oplus\left(\oplus_{i \in I} \mathbb{C} d_{i}\right)$ is called the Cartan subalgebra of $\mathfrak{g}$ and the linear functionals $\alpha_{i} \in \mathfrak{h}^{\star}(i \in I)$ defined by (2.1) are called the simple roots of $\mathfrak{g}$. For each $i \in I^{\text {re }}$, let $r_{i} \in G L\left(\mathfrak{h}^{\star}\right)$ be the simple reflection of $\mathfrak{h}^{\star}$ defined by

$$
r_{i}(\lambda)=\lambda-\lambda\left(h_{i}\right) \alpha_{i} \quad\left(\lambda \in \mathfrak{h}^{\star}\right) .
$$

The subgroup $W$ of $G L\left(\mathfrak{h}^{\star}\right)$ generated by the $r_{i}$ 's $\left(i \in I^{\mathrm{re}}\right)$ is called the Weyl group of the Borcherds superalgebra $\mathfrak{g}$.

The Borcherds superalgebra $\mathfrak{g}=\mathfrak{g}(A, \underline{m}, C)$ has the root space decomposition $\mathfrak{g}=\oplus_{\alpha \in Q} \mathfrak{g}_{\alpha}$, where

$$
\mathfrak{g}_{\alpha}=\{x \in \mathfrak{g} \mid[h, x]=\alpha(h) x \text { for all } h \in \mathfrak{h}\} .
$$

Note that

$$
\mathfrak{g}_{\alpha_{i}}=\mathbb{C} e_{i, 1} \oplus \cdots \oplus \mathbb{C} e_{i, m_{i}}
$$

and

$$
\mathfrak{g}_{-\alpha_{i}}=\mathbb{C} f_{i, 1} \oplus \cdots \oplus \mathbb{C} f_{i, m_{i}} .
$$

We say that $\alpha \in Q^{X}$ is a root of $\mathfrak{g}$ if $\mathfrak{g}_{\alpha} \neq 0$. The subspace $\mathfrak{g}_{\alpha}$ is called the root space of $\mathfrak{g}$ attached to $\alpha$. A root $\alpha$ is called real if $(\alpha \mid \alpha)>0$ and imaginary if $(\alpha \mid \alpha) \leq 0$.

In particular, a simple root $\alpha_{i}$ is real if $a_{i i}=2$ that is if $i \in I^{\mathrm{re}}$ and imaginary if $a_{i i} \leq 0$ that is if $i \in I^{\mathrm{im}}$. Note that the imaginary simple roots may have multiplicity $>1$. A root $\alpha>0(\alpha<0)$ is called positive (negative). 
One can show that all the roots are either positive or negative. We denote by $\Delta, \Delta_{+}$and $\Delta_{-}$the set of all roots, positive roots and negative roots, respectively. Also we denote $\Delta_{\overline{0}}\left(\Delta_{\overline{1}}\right)$ the set of all even (odd) roots of $\mathfrak{g}$.

Define the subspaces $\mathfrak{g}^{ \pm}=\oplus_{\alpha \in \Delta_{ \pm}} \mathfrak{g}_{\alpha}$.

Then we have the triangular decomposition of $\mathfrak{g}$ :

$$
\mathfrak{g}=\mathfrak{g}^{-} \oplus \mathfrak{h} \oplus \mathfrak{g}^{+} .
$$

Definition 2.8 (Sthanumoorthy et al. ([37])). We define an indefinite nonhyperbolic Borcherds-Cartan matrix $A$ to be of extended-hyperbolic type if every principal submatrix of $A$ is of finite, affine, or hyperbolic type BorcherdsCartan matrix. We say that the Borcherds superalgebra associated with a Borcherds-Cartan matrix $A$ is of extended-hyperbolic type, if $A$ is of extendedhyperbolic type.

Definition 2.9. A $\mathfrak{g}$-module $V$ is called $\mathfrak{h}$-diagonalizable if it admits a weight space decomposition $V=\bigoplus_{\mu \in \mathfrak{h}^{*}} V_{\mu}$, where

$$
V_{\mu}=\{v \in V \mid h \cdot v=\mu(h) v \text { for all } h \in \mathfrak{h}\} .
$$

If $V_{\mu} \neq 0$, then $\mu$ is called a weight of $V$, and $\operatorname{dim} V_{\mu}$ is called the multiplicity of $\mu$ in $V$.

Definition 2.10. A h-diagonalizable $\mathfrak{g}$-module $V$ is called a highest weight module with highest weight $\lambda \in \mathfrak{h}^{*}$, if there is a nonzero vector $v_{\lambda} \in V$ such that

(i) $e_{i k} \cdot v_{\lambda}=0$, for all $i \in I, k=1, \cdots, m_{i}$,

(ii) $h \cdot v_{\lambda}=\lambda(h) v_{\lambda}$ for all $h \in \mathfrak{h}$,

(iii) $V=U(\mathfrak{g}) \cdot v_{\lambda}$. The vector $v_{\lambda}$ is called a highest weight vector.

For a highest weight module $V$ with highest weight $\lambda$, we have

(i) $V=U\left(\mathfrak{g}^{-}\right) \cdot v_{\lambda}$,

(ii) $V=\oplus_{\mu \leq \lambda} V_{\mu}, V_{\lambda}=\mathbb{C} v_{\lambda}$ and

(iii) $\operatorname{dim} V_{\mu}<\infty$ for all $\mu \leq \lambda$.

Definition 2.11. Let $P(V)$ denote the set of all weights of $V$. When all the weights spaces are finite dimensional, the character of $V$ is defined to be

$$
\operatorname{ch} V=\sum_{\mu \in \mathfrak{h}^{\star}}\left(\operatorname{dim} V_{\mu}\right) e^{\mu},
$$

where $e^{\mu}$ are the basis elements of the group $\mathbb{C}\left[\mathfrak{h}^{\star}\right]$ with the multiplication given by $e^{\mu} e^{\nu}=e^{\mu+\nu}$ for $\mu, \nu \in \mathfrak{h}^{\star}$.

Let $b_{+}=\mathfrak{h} \oplus \mathfrak{g}_{+}$be the Borel subalgebra of $\mathfrak{g}$ and $\mathbb{C}_{\lambda}$ be the 1-dimensional $b_{+}$-module defined by $\mathfrak{g}_{+} \cdot 1=0, h \cdot 1=\lambda(h) 1$ for all $h \in \mathfrak{h}$. The induced module $M(\lambda)=U(\mathfrak{g}) \otimes_{U\left(b_{+}\right)} \mathbb{C}_{\lambda}$ is called the Verma module over $\mathfrak{g}$ with highest weight $\lambda$. Every highest weight $\mathfrak{g}$-module with highest weight $\lambda$ is a homomorphic 
image of $M(\lambda)$ and the Verma module $M(\lambda)$ contains a unique maximal submodule $J(\lambda)$. Hence the quotient $V(\lambda)=M(\lambda) / J(\lambda)$ is irreducible.

Let $P^{+}$be the set of all linear functionals $\lambda \in \mathfrak{h}^{\star}$ satisfying

$$
\begin{cases}\lambda\left(h_{i}\right) \in \mathbb{Z}_{\geq 0} & \text { for all } i \in I^{\mathrm{re}}, \\ \lambda\left(h_{i}\right) \in 2 \mathbb{Z}_{\geq 0} & \text { for all } i \in I^{\mathrm{re}} \cap I^{\text {odd }}, \\ \lambda\left(h_{i}\right) \geq 0 & \text { for all } i \in I^{\mathrm{im}} .\end{cases}
$$

The elements of $\mathrm{P}^{+}$are called the dominant integral weights.

Let $\rho \in \mathfrak{h}^{\star}$ be the $\mathbb{C}$-linear functional satisfying $\rho\left(h_{i}\right)=\frac{1}{2} a_{i i}$ for all $i \in I$. Let $T$ denote the set of all imaginary simple roots counted with multiplicities and, for $F \subset T$, we set $F \perp \lambda$ if $\lambda\left(h_{i}\right)=0$ for all $\alpha_{i} \in F$.

Definition 2.12 (Kang and Kim $([17])$ ). Let $J$ be a finite subset of $I^{\text {re }}$ and we denote by $\Delta_{J}=\Delta \cap\left(\sum_{j \in J} \mathbb{Z} \alpha_{j}\right), \Delta_{J}^{ \pm}=\Delta^{ \pm} \cap \Delta_{J}$ and $\Delta^{ \pm}(J)=$ $\Delta^{ \pm} \backslash \Delta_{J}^{ \pm}$. Let

$$
\mathfrak{g}_{0}^{(J)}=\mathfrak{h} \bigoplus\left(\bigoplus_{\alpha \in \Delta_{J}} \mathfrak{g}_{\alpha}\right)
$$

and

$$
\mathfrak{g}_{ \pm}^{(J)}=\bigoplus_{\alpha \in \Delta^{ \pm}(J)} \mathfrak{g}_{\alpha}
$$

Then $\mathfrak{g}_{0}^{(J)}$ is the restricted Kac-Moody superalgebra (with an extended Cartan subalgebra) associated with the Cartan matrix $A_{J}=\left(a_{i j}\right)_{i, j \in J}$ and the set of odd indices

$$
J^{\text {odd }}=J \cap I^{\text {odd }}=\left\{j \in J \mid c_{j j}=-1\right\} .
$$

Then the triangular decomposition of $\mathfrak{g}$ is given by

$$
\mathfrak{g}=\mathfrak{g}_{-}^{(J)} \bigoplus \mathfrak{g}_{0}^{(J)} \bigoplus \mathfrak{g}_{+}^{(J)}
$$

Let $W_{J}=\left\langle r_{j} \mid j \in J\right\rangle$ be the subgroup of $W$ generated by the simple reflections $r_{j}(j \in J)$, and let

$$
W(J)=\left\{w \in W \mid \Delta_{w} \subset \Delta^{+}(J)\right\}
$$

where

$$
\Delta_{w}=\left\{\alpha \in \Delta^{+} \mid w^{-1} \alpha<0\right\} .
$$

Therefore $W_{J}$ is the Weyl group of the restricted Kac-Moody super algebra $\mathfrak{g}_{0}^{(J)}$ and $W(J)$ is the set of right coset representatives of $W_{J}$ in $W$. That is $W=W_{J} W(J)$.

The following lemma given in Kang and Kim ([18]), proved in Liu ([22]), is very useful in actual computation of the elements of $W(J)$.

Lemma 2.13. Suppose $w=w^{\prime} r_{j}$ and $l(w)=l\left(w^{\prime}\right)+1$. Then $w \in W(J)$ if and only if $w^{\prime} \in W(J)$ and $w^{\prime}\left(\alpha_{j}\right) \in \Delta^{+}(J)$. 
Now, let $\Delta_{\bar{i}, J}^{ \pm}=\Delta_{\bar{i}}^{ \pm} \cap \Delta_{J}(i=0,1)$ and $\Delta_{\bar{i}}^{ \pm}(J)=\Delta_{\bar{i}}^{ \pm} \backslash \Delta_{\bar{i}, J}^{ \pm}(i=0,1)$. Here $\Delta_{\overline{0}}^{ \pm}\left(\Delta_{\overline{1}}^{ \pm}\right)$denotes the set of all positive or negative even (resp., positive or negative odd) roots of $\mathfrak{g}$.

The following proposition, proved in Kang and Kim ([17]), gives the denominator identity for Borcherds superalgebras.

Proposition 2.14. Let $J$ be a finite subset of the set of all real indices $I^{\mathrm{re}}$. Then

$$
\frac{\prod_{\alpha \in \Delta_{\overline{0}(J)}^{-}}\left(1-e^{\alpha}\right)^{\operatorname{dim} \mathfrak{g}_{\alpha}}}{\prod_{\alpha \in \Delta_{\overline{\bar{T}}(J)}}\left(1+e^{\alpha}\right)^{\operatorname{dim} \mathfrak{g}_{\alpha}}}=\sum_{\substack{w \in W(J) \\ F \subset T}}(-1)^{l(w)+|F|} \operatorname{ch} V_{J}(w(\rho-s(F)-\rho)),
$$

where $V_{J}(\mu)$ denotes the irreducible highest weight module over the restricted Kac-Moody superalgebra $\mathfrak{g}_{0}^{(J)}$ with highest weight $\mu$ and where $F$ runs over all the finite subsets of $T$ such that any two elements of $F$ are mutually perpendicular. Here $l(w)$ denotes the length of $w,|F|$ the number of elements in $F$, and $s(F)$ the sum of the elements in $F$.

Definition 2.15. A basis elements of the group algebra $\mathbb{C}\left[\mathfrak{h}^{\star}\right]$ by defining

$$
E^{\alpha}=\theta(\alpha, \alpha) e^{\alpha} .
$$

Also define the super dimension $\operatorname{Dim} \mathfrak{g}_{\alpha}$ of the root space $\mathfrak{g}_{\alpha}$ by

$$
\operatorname{Dim} \mathfrak{g}_{\alpha}=\theta(\alpha, \alpha) \operatorname{dim} \mathfrak{g}_{\alpha} .
$$

Since $w(\rho-s(F))-\rho$ is an element of $Q_{-}$, all the weights of the irreducible highest weight $\mathfrak{g}_{0}^{(J)}$-module $V_{J}(w(\rho-s(F))-\rho)$ are also elements of $Q_{-}$.

Hence one can define the superdimension $\operatorname{Dim} V_{\mu}$ of the weight space $V_{\mu}$ of $V_{J}(w(\rho-s(F))-\rho)$ in a similar way. More generally, for an $\mathfrak{h}$-diagonalizable $\mathfrak{g}_{0}^{(J)}$-module $V=\oplus_{\mu \in \mathfrak{h}^{\star}} V_{\mu}$ such that $P(V) \subset Q$, we define the superdimension Dim $V_{\mu}$ of the weight space $V_{\mu}$ to be

$$
\operatorname{Dim} V_{\mu}=\theta(\mu, \mu) \operatorname{dim} V_{\mu}
$$

For each $k \geq 1$, let

$$
H_{k}^{(J)}=\bigoplus_{\substack{w \in W(J) \\ F \subset T \\ l(w)+|F|=k}} V_{J}(w(\rho-s(F))-\rho)
$$

and define the homology space $H^{(J)}$ of $\mathfrak{g}_{-}^{(J)}$ to be

$$
H^{(J)}=\sum_{k=1}^{\infty}(-1)^{k+1} H_{k}^{(J)}=H_{1}^{(J)} \ominus H_{2}^{(J)} \oplus H_{3}^{(J)} \ominus \cdots,
$$

an alternating direct sum of the vector spaces. 
For $\tau \in Q_{-}$, define the superdimension $\operatorname{Dim} H_{\tau}^{(J)}$ of the $\tau$-weight space of $H^{(J)}$ to be

$$
\begin{aligned}
\operatorname{Dim} H_{\tau}^{(J)} & =\sum_{k=1}^{\infty}(-1)^{k+1}\left(\operatorname{Dim} H_{k}^{(J)}\right)_{\tau} \\
& =\sum_{k=1}^{\infty}(-1)^{k+1} \sum_{\substack{w \in W(J) \\
F \subset T \\
l(w)+|F|=k}} \operatorname{Dim} V_{J}(w(\rho-s(F))-\rho)_{\tau},
\end{aligned}
$$

$$
\operatorname{Dim} H_{\tau}^{(J)}=\sum_{\substack{w \in W(J) \\ F \subset T \\ l(w)+|F| \geq 1}}(-1)^{l(w)+|F|+1} \operatorname{Dim} V_{J}(w(\rho-s(F))-\rho)_{\tau} .
$$

Let

$$
P\left(H^{(J)}\right)=\left\{\alpha \in Q^{-}(J) \mid \operatorname{dim} H_{\alpha}^{(J)} \neq 0\right\}
$$

and let $\left\{\tau_{1}, \tau_{2}, \tau_{3}, \cdots\right\}$, be an enumeration of the set $P\left(H^{(J)}\right)$. Let $D(i)=$ $\operatorname{Dim} H_{\tau_{i}}^{(J)}$.

REMARK 2.16. The elements of $P\left(H^{(J)}\right)$ can be determined by applying the following proposition, proved in Kac $([8])$.

Proposition 2.17 (Kang and $\operatorname{Kim}([17]))$. Let $\Lambda \in P_{+}$. Then $P(\Lambda)=$ $W .\left\{\lambda \in P_{+} \mid \lambda\right.$ is nondegenerate with respect to $\left.\Lambda\right\}$.

Now, for $\tau \in Q^{-}(J)$, define

$$
T^{(J)}(\tau)=\left\{n=\left(n_{i}\right)_{i \geq 1} \mid n_{i} \in \mathbb{Z}_{\geq 0}, \sum n_{i} \tau_{i}=\tau\right\},
$$

the set of all partitions of $\tau$ into a sum of $\alpha_{i}$ 's.

For $n \in T^{(J)}(\alpha)$, use the notations $|n|=\sum n_{i}$ and $n !=\prod n_{i}$ !.

Now, for $\tau \in Q^{-}(J)$, the Witt partition function $W^{(J)}(\tau)$ is defined as

$$
W^{(J)}(\tau)=\sum_{n \in T^{(J)}(\tau)} \frac{(|n|-1) !}{n !} \prod D(i)^{n_{i}} .
$$

The proof of a closed form formula for the superdimension Dim $\mathfrak{g}_{\alpha}$ of the root space $\mathfrak{g}_{\alpha}\left(\alpha \in \Delta^{-}(J)\right)$ is given in the following theorem of Kang and Kim $([17])$.

TheOREm 2.18 (Kang and Kim $([17]))$. Let $J$ be a finite subset of $I^{\mathrm{re}}$. Then, for $\alpha \in \Delta^{-}(J)$, we have

$$
\operatorname{Dim} \mathfrak{g}_{\alpha}=\sum_{d \mid \alpha} \frac{1}{d} \mu(d) W^{(J)}\left(\frac{\alpha}{d}\right),
$$




$$
\operatorname{Dim} \mathfrak{g}_{\alpha}=\sum_{d \mid \alpha} \frac{1}{d} \mu(d) \sum_{n \in T^{(J)}\left(\frac{\alpha}{d}\right)} \frac{(|n|-1) !}{n !} \prod D(i)^{n_{i}}
$$

where $\mu$ is the classical Möbius function. Namely, for a natural number n, $\mu(n)$ is defined as follows:

$$
\mu(n)= \begin{cases}1 & \text { for } n=1 \\ (-1)^{k} & \text { for } n=p_{1} \cdots p_{k}\left(p_{1}, \cdots, p_{k}: \text { distinct primes }\right), \\ 0 & \text { if it is not square free }\end{cases}
$$

and, for a positive integer $d, d \mid \alpha$ denotes $\alpha=d \alpha$ for some $\alpha \in Q_{-}$, in which case $\alpha=\frac{\alpha}{d}$.

In the following Sections 3.1, 3.2 and 3.3, we find root supermultiplicities of Borcherds superalgebras which are the extensions of Kac-Moody Algebras $A_{2}$ and $A_{3}$ with multiplicity greater than or equal 1 and the corresponding combinatorial identities using Kang and Kim ([17]). In Section 3.4, we also find superdimension formula for two Borcherds superalgebras which are extensions of hyperbolic Kac-Moody algebras.

\section{Root SUPERMUltiplicities (WITH MULTIPLICITY $\geq 1$ ) OF BorChERDS SUPERALGEBRAS WHICH ARE THE EXTENSIONS OF KAC MOODY ALGEBRAS AND THE CORRESPONDING COMBINATORIAL IDENTITIES}

3.1. Superdimension formula and the corresponding combinatorial identity for the extended-hyperbolic Borcherds superalgebra which is an extension of $A_{2}$. We are considering $J$ as two different subsets of $I^{r e}$ and getting the following dimension formula and combinatorial identity for $\alpha=\tau=-4 \alpha_{1}-$ $2 \alpha_{2}-3 \alpha_{3} \in Q_{-}$with $\mathrm{b}=1$ for the extended-hyperbolic Borcherds superalgebra $\mathfrak{g}=\mathfrak{g}(A, \underline{m}, C)$ associated with the extended-hyperbolic Borcherds-Cartan super matrix

$$
A=\left(\begin{array}{ccc}
-k & -a & -b \\
-a & 2 & -1 \\
-b & -1 & 2
\end{array}\right)
$$

Consider the extended-hyperbolic Borcherds superalgebra $\mathfrak{g}=\mathfrak{g}(A, \underline{m}, C)$ associated with the extended-hyperbolic Borcherds-Cartan super matrix

$$
A=\left(\begin{array}{ccc}
-k & -a & -b \\
-a & 2 & -1 \\
-b & -1 & 2
\end{array}\right)
$$

and the corresponding coloring matrix be

$$
C=\left(\begin{array}{ccc}
-1 & c_{1} & c_{2} \\
c_{1}^{-1} & 1 & c_{3} \\
c_{2}^{-1} & c_{3}^{-1} & 1
\end{array}\right)
$$


with $c_{1}, c_{2}, c_{3} \in C^{X}$.

Let $I=\{1,2,3\}$ be the index set with charge $\underline{m}=\{1,1,1\}$.

Let us consider the root $\alpha=k_{1} \alpha_{1}+k_{2} \alpha_{2}+k_{3} \alpha_{3} \in Q$. Then we have $\theta(\alpha, \alpha)=(-1)^{k_{1}^{2}}$. Hence $\alpha$ is an even root (resp. odd root) if $k_{1}$ is even integer (resp. odd integer). Also $T=\left\{\alpha_{1}\right\}$ and the subset $F \subset T$ is either empty or $\left\{\alpha_{1}\right\}$. Take $J \subseteq I^{r e}$ as $J=\{2\}$. By Lemma 2.13, this implies that $W(J)=\left\{1, r_{3}\right\}$. From equations (2.7) and (2.8), the homological space can be written as

$$
\begin{aligned}
H_{1}^{(J)} & =V_{J}\left(1\left(\rho-\alpha_{1}\right)-\rho\right) \oplus V_{J}\left(r_{3}(\rho)-\rho\right) \\
& =V_{J}\left(-\alpha_{1}\right) \oplus V_{J}\left(-\alpha_{3}\right), \\
H_{2}^{(J)} & =V_{J}\left(r_{3}\left(\rho-\alpha_{1}\right)-\rho\right)=V_{J}\left(-\alpha_{1}-(b+1) \alpha_{3}\right), \\
H_{3}^{(J)} & =0 \text { for all } k \geq 3
\end{aligned}
$$

and hence

$$
\begin{aligned}
H^{(J)} & =H_{1}^{(J)} \ominus H_{2}^{(J)} \\
& =V_{J}\left(-\alpha_{1}\right) \oplus V_{J}\left(-\alpha_{3}\right) \ominus V_{J}\left(-\alpha_{1}-(b+1) \alpha_{3}\right),
\end{aligned}
$$

with

$$
\begin{aligned}
& \operatorname{Dim} H_{(1,0,0)}^{(J)}=-1 \neq 0 ; \quad \operatorname{Dim} H_{(0,1,0)}^{(J)}=-1 \neq 0 ; \\
& \operatorname{Dim} H_{(0,0,1)}^{(J)}=1 \neq 0 ; \quad \operatorname{Dim} H_{(1,1, b+1)}^{(J)}=-1 \neq 0 .
\end{aligned}
$$

So we have

$$
P\left(H^{(J)}\right)=\{(1,0,0),(0,1,0),(0,0,1),(1,1, b+1)\} .
$$

Let $\tau=\alpha=-p \alpha_{1}-q \alpha_{2}-t \alpha_{3} \in Q_{-}$, with $(p, q, t) \in \mathbb{Z}_{\geq 0} \times \mathbb{Z}_{\geq 0} \times \mathbb{Z}_{\geq 0}$. Then by Proposition 2.17 we get

$$
\begin{gathered}
T^{(J)}(\tau)=\left\{\left(s_{1}, s_{2}, s_{3}, s_{4}\right) \mid s_{1}(1,0,0)+s_{2}(0,1,0)+s_{3}(0,0,1)+s_{4}(1,1, b+1)\right. \\
=(p, q, t)\} .
\end{gathered}
$$

This implies

$$
\begin{aligned}
s_{1}+s_{4} & =p, \\
s_{2}+s_{4} & =q, \\
s_{3}+(b+1) s_{4} & =t .
\end{aligned}
$$

So, we have

$$
s_{1}=p-s_{4} ; s_{2}=q-s_{4} ; s_{3}=t-(b+1) s_{4} ; s_{4}=0 \text { to } \min \left(p, q,\left[\frac{t}{b+1}\right]\right) .
$$


Applying $s_{1}, s_{2}, s_{3}, s_{4}$ in Witt partition formula (2.12), we get

$$
W^{(J)}(\tau)=\sum_{s_{4}=0}^{\min \left(p, q,\left[\frac{t}{b+1}\right]\right)} \frac{\left(p+q+t-(b+2) s_{4}-1\right) !(-1)^{p+q-s_{4}}}{\left(p-s_{4}\right) !\left(q-s_{4}\right) !\left(t-(b+1) s_{4}\right) ! s_{4} !}
$$

From (2.13), the dimension of $\mathfrak{g}_{\alpha}$ is

$$
\begin{aligned}
\operatorname{Dim} \mathfrak{g}_{\alpha} & =\sum_{d \mid \alpha} \frac{1}{d} \mu(d) W^{(J)}\left(\frac{\alpha}{d}\right) \\
& =\sum_{d \mid \alpha} \frac{1}{d} \mu(d) \sum_{n \in T^{(J)}\left(\frac{\alpha}{d}\right)} \frac{(|n|-1) !}{n !} \prod D(i)^{n_{i}},
\end{aligned}
$$

Substituting the value of $W^{(J)}(\tau)$ from (3.1) in the above dimension formula, we get

$\operatorname{Dim} \mathfrak{g}_{\alpha}=\sum_{d \mid \alpha} \frac{1}{d} \mu(d) \sum_{s_{4}=0}^{\min \left(\frac{p}{d}, \frac{q}{d},\left[\frac{t}{d(b+1)}\right]\right)} \frac{\left(\frac{p}{d}+\frac{q}{d}+\frac{t}{d}-(b+2) s_{4}-1\right) !(-1)^{\frac{p}{d}+\frac{q}{d}-s_{4}}}{\left(\frac{p}{d}-s_{4}\right) !\left(\frac{q}{d}-s_{4}\right) !\left(\frac{t}{d}-(b+1) s_{4}\right) ! s_{4} !}$.

On the other hand if we consider, $\{J\}=\{2,3\}$. Then $W(J)=\{1\}$ and $\mathfrak{g}_{0}^{(J)}=\left\langle e_{1}, f_{1}, h\right\rangle \cong \operatorname{sl}(2, \mathbb{C})$ and $T=\left\{\alpha_{1}\right\} . F \subset T$ is either empty or $\left\{\alpha_{1}\right\}$. Using (2.7) and (2.8), we have $H^{(J)}=V_{J}\left(-\alpha_{1}\right)$. We consider $H^{(J)}$ as a 4 dimensional irreducible representation of the Lie algebra $\operatorname{sl}(2, \mathbb{C})$ and hence we obtain

$$
P\left(H^{(J)}\right)=\{(1,0,1),(0,1,1),(1,1,1),(1,1,2)\} .
$$

with

$$
\begin{array}{ll}
\operatorname{Dim} H_{(1,0,1)}^{(J)}=-1 ; & \operatorname{Dim} H_{(0,1,1)}^{(J)}=-1 ; \\
\operatorname{Dim} H_{(1,1,1)}^{(J)}=-1 ; & \operatorname{Dim} H_{(1,1,2)}^{(J)}=-1 .
\end{array}
$$

Let $\tau=-p \alpha_{1}-q \alpha_{2}-t \alpha_{3} \in Q_{-}$, with $(p, q, t) \in \mathbb{Z}_{\geq 0} \times \mathbb{Z}_{\geq 0} \times \mathbb{Z}_{\geq 0}$. Then by Proposition 2.17 we get

$$
\begin{gathered}
T^{(J)}(\tau)=\left\{\left(s_{1}, s_{2}, s_{3}, s_{4}\right) \mid\right. \\
\left(p-\left|\phi_{1}\right|, q-\left|\phi_{2}\right|,\left(\phi_{1}, \phi_{2}\right)\right), p-\left|\phi_{1}\right|=1,2, \ldots, \\
q-\left|\phi_{2}\right|=0,1,2, \ldots, \\
\phi_{1} \text { is partition of } q \text { with parts } \leq 2 ; \\
\left.\phi_{2} \text { is partition of } t \text { with parts } \leq 2\right\} .
\end{gathered}
$$

Applying $s_{1}, s_{2}, s_{3}, s_{4}$ in Witt partition formula, we get

$$
W^{(J)}(\tau)=\sum_{\phi \in T^{(J)}(\alpha)} \frac{(p-1) !(-1)^{p+q}}{\left(p-\left|\phi_{1}\right|\right) !\left(q-\left|\phi_{2}\right|\right) ! \phi_{1} ! \phi_{2} !} .
$$


From (2.13), the dimension of $\mathfrak{g}_{\alpha}$ is

$$
\begin{aligned}
\operatorname{Dim} \mathfrak{g}_{\alpha} & =\sum_{d \mid \alpha} \frac{1}{d} \mu(d) W^{(J)}\left(\frac{\alpha}{d}\right) \\
& =\sum_{d \mid \alpha} \frac{1}{d} \mu(d) \sum_{n \in T^{(J)}\left(\frac{\alpha}{d}\right)} \frac{(|n|-1) !}{n !} \prod D(i)^{n_{i}}
\end{aligned}
$$

Substituting the value of $W^{(J)}(\tau)$ from (3.2) in the above dimension formula, we get

$$
\operatorname{Dim} \mathfrak{g}_{\alpha}=\sum_{d \mid \alpha} \frac{1}{d} \mu(d) \sum_{\phi \in T^{(J)}(\alpha)} \frac{(p-1) !(-1)^{p+q}}{\left(p-\left|\phi_{1}\right|\right) !\left(q-\left|\phi_{2}\right|\right) ! \phi_{1} ! \phi_{2} !}
$$

Now let us consider Borcherds-Cartan matrix

$$
A=\left(\begin{array}{ccc}
-k & -a & -1 \\
-a & 2 & -1 \\
-1 & -1 & 2
\end{array}\right)
$$

(that is $b=1$ in the above Borcherds-Cartan matrix). For this BorcherdsCartan matrix, we consider the root $\tau=\alpha=(4,2,3) \in Q_{-}$.

Substituting $\tau=\alpha=(4,2,3) \in Q_{-}$and $b=1$ in (3.1), we have

$$
\begin{aligned}
W^{(J)}(\tau) & =\sum_{s_{4}=0}^{\min \left(p, q,\left[\frac{t}{b+1}\right]\right)} \frac{\left(p+q+t-(b+2) s_{4}-1\right) !(-1)^{p+q-s_{4}}}{\left(p-s_{4}\right) !\left(q-s_{4}\right) !\left(t-(b+1) s_{4}\right) ! s_{4} !} \\
& =\sum_{s_{4}=0}^{\min \left(4,2,\left[\frac{3}{2}\right]\right)} \frac{\left(4+2+3-3 s_{4}-1\right) !(-1)^{6-s_{4}}}{\left(4-s_{4}\right) !\left(2-s_{4}\right) !\left(3-2 s_{4}\right) ! s_{4} !} \\
& =\frac{8 !(-1)^{6}}{4 ! 2 ! 3 !}+\frac{5 !(-1)^{5}}{3 ! 1 ! 1 ! 1 !}=120 .
\end{aligned}
$$

Substituting the root $(4,2,3)$ and $b=1$ in $(3.2)$, we have

$$
\begin{aligned}
W^{(J)}(\tau) & =\sum_{\phi \in T^{(J)}(\tau)} \frac{(p+q-1) !(-1)^{p+q}}{\left(p-\left|\phi_{1}\right|\right) !\left(q-\left|\phi_{2}\right|\right) ! \phi_{1} ! \phi_{2} !} \\
& =\frac{5 !}{2 ! 1 ! 1 ! 1 !}+\frac{5 !}{2 ! 2 !}+\frac{5 !}{3 !}+\frac{5 !}{3 ! 2 !} \\
& =120 .
\end{aligned}
$$


Hence the combinatorial identity

$$
\begin{aligned}
\sum_{s_{4}=0}^{\min \left(p, q,\left[\frac{t}{b+1}\right]\right)} & \frac{\left(p+q+t-(b+2) s_{4}-1\right) !(-1)^{p+q-s_{4}}}{\left(p-s_{4}\right) !\left(q-s_{4}\right) !\left(t-(b+1) s_{4}\right) ! s_{4} !} \\
= & \sum_{\phi \in T^{(J)}(\tau)} \frac{(p+q-1) !(-1)^{p+q}}{\left(p-\left|\phi_{1}\right|\right) !\left(q-\left|\phi_{2}\right|\right) ! \phi_{1} ! \phi_{2} !}
\end{aligned}
$$

holds for the particular root $\tau=\alpha=-4 \alpha_{1}-2 \alpha_{2}-3 \alpha_{3} \in Q_{-}$for the Borcherds-Cartan matrix

$$
A=\left(\begin{array}{ccc}
-k & -a & -1 \\
-a & 2 & -1 \\
-1 & -1 & 2
\end{array}\right)
$$

Hence we have proved the following theorem:

TheOREM 3.1. Let $\mathfrak{g}=\mathfrak{g}(A, \underline{m}, C)$ be the extended-hyperbolic Borcherds superalgebra associated with the extended-hyperbolic Borcherds-Cartan super matrix

$$
A=\left(\begin{array}{ccc}
-k & -a & -b \\
-a & 2 & -1 \\
-b & -1 & 2
\end{array}\right)
$$

with charge $\underline{m}=\{1,1,1\}$. Then for the root $\tau=\alpha=-p \alpha_{1}-q \alpha_{2}-t \alpha_{3} \in Q_{-}$, the dimension of $\mathfrak{g}_{\alpha}$ is

$\operatorname{Dim} \mathfrak{g}_{\alpha}=\sum_{d \mid \alpha} \frac{1}{d} \mu(d) \sum_{s_{4}=0}^{\min \left(\frac{p}{d}, \frac{q}{d},\left[\frac{t}{d(b+1)}\right]\right)} \frac{\left(\frac{p}{d}+\frac{q}{d}+\frac{t}{d}-(b+2) s_{4}-1\right) !(-1)^{\frac{p}{d}+\frac{q}{d}-s_{4}}}{\left(\frac{p}{d}-s_{4}\right) !\left(\frac{q}{d}-s_{4}\right) !\left(\frac{t}{d}-(b+1) s_{4}\right) ! s_{4} !}$

or

$$
\operatorname{Dim} \mathfrak{g}_{\alpha}=\sum_{d \mid \alpha} \frac{1}{d} \mu(d) \sum_{\phi \in T^{(J)}(\tau)} \frac{(p-1) !(-1)^{p+q}}{\left(p-\left|\phi_{1}\right|\right) !\left(q-\left|\phi_{2}\right|\right) ! \phi_{1} ! \phi_{2} !}
$$

for different values of $J \subseteq I^{\text {re }}$.

Moreover, for a particular root $\tau=\alpha=-4 \alpha_{1}-2 \alpha_{2}-3 \alpha_{3}$, the following combinatorial identity holds:

$$
\begin{aligned}
\sum_{s_{4}=0}^{\min \left(p, q,\left[\frac{t}{b+1}\right]\right)} & \frac{\left(p+q+t-(b+2) s_{4}-1\right) !(-1)^{p+q-s_{4}}}{\left(p-s_{4}\right) !\left(q-s_{4}\right) !\left(t-(b+1) s_{4}\right) ! s_{4} !} \\
= & \sum_{\phi \in T^{(J)}(\tau)} \frac{(p+q-1) !(-1)^{p+q}}{\left(p-\left|\phi_{1}\right|\right) !\left(q-\left|\phi_{2}\right|\right) ! \phi_{1} ! \phi_{2} !}
\end{aligned}
$$

where $\phi_{1}$ is partition of ' $q$ ' with parts $\leq 2$ and $\phi_{2}$ is partition of ' $t$ ' with parts $\leq 2$. 
REMARK 3.2. If $a=1, b=0$, the above example is same as the example

$$
A=\left(\begin{array}{ccc}
-k & -a & -b \\
-a & 2 & -1 \\
-b & -1 & 2
\end{array}\right)
$$

given in Sthanumoorthy et al. ([36]). But in this present case, we consider two different cases of $J \subseteq I^{r e}$ and found the corresponding Dimension formulas. Although, the equality of above two values of Dim $\mathfrak{g}_{\alpha}$ is not proved here in general, the same is proved for a particular root $\alpha=(4,2,3)$ and $b=1$ in the Borcherds-Cartan super matrix

$$
A=\left(\begin{array}{ccc}
-k & -a & -b \\
-a & 2 & -1 \\
-b & -1 & 2
\end{array}\right)
$$

Below, we find the dimension formulae and combinatorial identities for the Borcherds superalgebras which are extensions of $A_{2}, A_{3}$ (for a same set $J \subseteq I^{r e}$ ) by solving $T^{(J)}(\tau)$ in two different method.

3.2. Superdimension formula and the corresponding combinatorial identity for the extended-hyperbolic Borcherds superalgebra which is an extension of $A_{2}$. Consider the extended-hyperbolic Borcherds superalgebra $\mathfrak{g}=\mathfrak{g}(A, \underline{m}, C)$ associated with the extended-hyperbolic Borcherds-Cartan super matrix

$$
A=\left(\begin{array}{ccc}
-k & -a & -b \\
-a & 2 & -1 \\
-b & -1 & 2
\end{array}\right)
$$

and the corresponding coloring matrix be

$$
C=\left(\begin{array}{ccc}
-1 & c_{1} & c_{2} \\
c_{1}^{-1} & 1 & c_{3} \\
c_{2}^{-1} & c_{3}^{-1} & 1
\end{array}\right)
$$

with $c_{1}, c_{2}, c_{3} \in C^{X}$.

Let $I=\{1,2,3\}$ be the index set for the simple roots of $\mathfrak{g}$. Here $\alpha_{1}$ is the imaginary odd simple root with multiplicity $r \geq 1$ and $\alpha_{2}, \alpha_{3}$ are the real even simple roots.

Let us consider the root $\alpha=k_{1} \alpha_{1}+k_{2} \alpha_{2}+k_{3} \alpha_{3} \in Q$. We have $\theta(\alpha, \alpha)=$ $(-1)^{k_{1}^{2}}$. Hence $\alpha$ is an even root (resp. odd root) if $k_{1}$ is even integer (resp. odd integer). Also $T=\left\{\alpha_{1}\right\}$ (counted $\mathrm{r}$ times) and the subset $F \subset T$ is either empty or $\left\{\alpha_{1}\right\}$. Take $J \subseteq I^{r e}$ as $J=\{2,3\}$. Then

$$
\mathfrak{g}_{0}=\left\langle e_{2}, f_{2}, h_{2}, e_{3}, f_{3}, h_{3}\right\rangle \cong \operatorname{sl}(3, \mathbb{C}) .
$$


By Lemma 2.13, this implies that $W(J)=\{1\}$. From (2.7) and (2.8), the homology space can be written as

$$
\begin{aligned}
& H_{1}^{(J)}=V_{J}\left(1\left(\rho-\alpha_{1}\right)-\rho\right)=V_{J}\left(-\alpha_{1}\right), \\
& H_{k}^{(J)}=0 \text { for all } k \geq 2 .
\end{aligned}
$$

Therefore

$$
\left.H^{(J)}=H_{1}^{(J)}=V_{J}\left(-\alpha_{1}\right) \oplus V_{J}\left(-\alpha_{1}\right) \oplus \cdots \oplus V_{J}\left(-\alpha_{1}\right) \quad \text { (counted } r \text { times }\right)
$$

with

$$
\begin{array}{ll}
\operatorname{Dim} H_{(1,0,0)}^{(J)}=-r, & \operatorname{Dim} H_{(1,1,0)}^{(J)}=-r \\
\operatorname{Dim} H_{(1,1,1)}^{(J)}=-r, & \operatorname{Dim} H_{(1,1, a+1)}^{(J)}=-r .
\end{array}
$$

We take

$$
P\left(H^{(J)}\right)=\{(1,0,0),(1,1,0),(1,1,1),(1,1, a+1)\} .
$$

Let $\tau=\alpha=-p \alpha_{1}-q \alpha_{2}-t \alpha_{3} \in Q_{-}$, with $(p, q, t) \in \mathbb{Z}_{\geq 0} \times \mathbb{Z}_{\geq 0} \times \mathbb{Z}_{\geq 0}$. Then by Proposition 2.17 we get

$$
\begin{gathered}
T^{(J)}(\tau)=\left\{\left(s_{1}, s_{2}, s_{3}, s_{4}\right) \mid s_{1}(1,0,0)+s_{2}(1,1,0)+s_{3}(1,1,1)+s_{4}(1,1, a+1)\right. \\
=(p, q, t)\}
\end{gathered}
$$

This implies

$$
\begin{aligned}
s_{1}+s_{2}+s_{3}+s_{4} & =p, \\
s_{2}+s_{3}+s_{4} & =q, \\
s_{3}+(a+1) s_{4} & =t .
\end{aligned}
$$

We have

$$
\begin{aligned}
& s_{1}=p-q \\
& s_{2}=q-t+a s_{4} \\
& s_{3}=t-(a+1) s_{4}, \\
& s_{4}=0 \text { to } \min \left(p, q,\left[\frac{t}{a+1}\right]\right) .
\end{aligned}
$$

Applying $s_{1}, s_{2}, s_{3}, s_{4}$ in Witt partition formula (2.12), we have

$$
W^{(J)}(\tau)=\sum_{\substack{s_{4}=0 \\\left(q-t+a s_{4}\right) \geq 0}}^{\min \left(p, q,\left[\frac{t}{a+1}\right]\right)} \frac{(p-1) !(-r)^{p}}{(p-q) !\left(q-t+a s_{4}\right) !\left(t-(a+1) s_{4}\right) ! s_{4} !}
$$

(note that $(p-q) \geq 0$ and $\left.\left(t-(a+1) s_{4}\right) \geq 0\right)$. 
From (2.13) we conclude that the dimension of $\mathfrak{g}_{\alpha}$ is

$$
\begin{aligned}
\operatorname{Dim} \mathfrak{g}_{\alpha} & =\sum_{d \mid \alpha} \frac{1}{d} \mu(d) W^{(J)}\left(\frac{\alpha}{d}\right) \\
& =\sum_{d \mid \alpha} \frac{1}{d} \mu(d) \sum_{n \in T^{(J)}\left(\frac{\alpha}{d}\right)} \frac{(|n|-1) !}{n !} \prod D(i)^{n_{i}},
\end{aligned}
$$

Substituting the value of $W^{(J)}(\tau)$ from (3.4) in the above dimension formula, we have

$$
\operatorname{Dim} \mathfrak{g}_{\alpha}=\sum_{\substack{s_{4}=0 \\\left(q-t+a s_{4}\right) \geq 0}}^{\min \left(p, q,\left[\frac{t}{a+1}\right]\right)} \frac{(p-1) !(-r)^{p}}{(p-q) !\left(q-t+a s_{4}\right) !\left(t-(a+1) s_{4}\right) ! s_{4} !}
$$

(note that $(p-q) \geq 0$ and $\left.\left(t-(a+1) s_{4}\right) \geq 0\right)$.

If we solve the same $P\left(H^{(J)}\right)$, using partition and substituting this partition in $T^{(J)}(\tau)$, we have

$$
T^{(J)}(\tau)=\{(p-q), q-|\phi|, \phi\},
$$

where $\phi$ is partition of $t$ with parts $(1, a+1)$ of length $t$. Applying $T^{(J)}(\tau)$ in Witt partition formula (2.12), we have

$$
W^{(J)}(\tau)=\sum_{\phi \in T^{(J)}(\tau)} \frac{(p-1) !(-r)^{p}}{(p-q) !(q-|\phi|) ! \phi !} .
$$

From (2.13), the dimension of $\mathfrak{g}_{\alpha}$ is

$$
\begin{aligned}
\operatorname{Dim} \mathfrak{g}_{\alpha} & =\sum_{d \mid \alpha} \frac{1}{d} \mu(d) W^{(J)}\left(\frac{\alpha}{d}\right) \\
& =\sum_{d \mid \alpha} \frac{1}{d} \mu(d) \sum_{n \in T^{(J)}\left(\frac{\alpha}{d}\right)} \frac{(|n|-1) !}{n !} \prod D(i)^{n_{i}},
\end{aligned}
$$

Substituting the value of $W^{(J)}(\tau)$ from (3.5) in the above dimension formula, we have

$$
\operatorname{Dim} \mathfrak{g}_{\alpha}=\sum_{\phi \in T^{(J)}(\tau)} \frac{(p-1) !(-r)^{p}}{(p-q) !(q-|\phi|) ! \phi !} .
$$

Consider (3.5)

$$
W^{(J)}(\tau)=\sum_{\phi \in T^{(J)}(\tau)} \frac{(p-1) !(-r)^{p}}{(p-q) !(q-|\phi|) ! \phi !}
$$

where $\phi$ is partitions of $t$ in $\alpha=\tau=-p \alpha_{1}-q \alpha_{2}-t \alpha_{3}$ with parts $(1, \mathrm{a}+1)$ of length $\leq t$. That is, $\phi$ can be written as $\left(n_{1}, n_{(a+1)}\right)$, where $n_{1}$ is number of 
1's in the partition of $t$ and $n_{(a+1)}$ is number of $(\mathrm{a}+1)$ 's in the same partition of $t$. Then

$\phi=\left\{(t, 0),(t-(a+1), 1), \ldots\right.$ up to the term satisfying $\left.t-n_{1}(a+1)>0\right\}$.

Hence

$$
\begin{aligned}
W^{(J)}(\tau)= & \sum_{\left(n_{1}, n_{(a+1)}\right) \in T^{(J)}(\tau)} \frac{(p-1) !(-r)^{p}}{(p-q) !\left(q-n_{1}-n_{(a+1)}\right) ! n_{1} ! n_{(a+1)} !} \\
= & \frac{(p-1) !(-r)^{p}}{(p-q) !(q-t-0) ! t ! 0 !} \\
& +\frac{(p-1) !(-r)^{p}}{(p-q) !(q-t+a+1-1) !(t-(a+1) 1) ! 1 !} \\
& +\frac{(p-1) !(-r)^{p}}{(p-q) !(q-t+2 a+2-2) !(t-2(a+1)) ! 2 !} \\
& +\cdots \text { up to the term satisfying } t-n_{1}(a+1)>0 \\
= & \frac{(p-1) !(-r)^{p}}{(p-q) !(q-t-0) ! t ! 0 !}+\frac{(p-1) !(-r)^{p}}{(p-q) !(q-t+a) !(t-(a+1)) ! 1 !} \\
& +\frac{(p-1) !(-r)^{p}}{(p-q) !(q-t+2 a) !(t-2(a+1)) ! 2 !} \\
& +\cdots \text { up to the term satisfying } t-n_{1}(a+1)>0 .
\end{aligned}
$$

Here we are considering the roots of type $(p, q, t)$ such that $p \geq q \leq t$ and also $p \geq t$. Moreover, the sum ranges up to the term satisfying $t-n_{1}(a+1)>0$. So we can write the above summation as

$$
\operatorname{Dim} \mathfrak{g}_{\alpha}=\sum_{\substack{s_{4}=0 \\\left(q-t+a s_{4}\right) \geq 0}}^{\min \left(p, q,\left[\frac{t}{a+1}\right]\right)} \frac{(p-1) !(-r)^{p}}{(p-q) !\left(q-t+a s_{4}\right) !\left(t-(a+1) s_{4}\right) ! s_{4} !},
$$

which is same as (3.4) (note that $(p-q) \geq 0$ and $\left(t-(a+1) s_{4}\right) \geq 0$ ).

Hence, we get the following theorem:

Theorem 3.3. Let $\mathfrak{g}=\mathfrak{g}(A, \underline{m}, C)$ be the extended-hyperbolic Borcherds superalgebra associated with the extended-hyperbolic Borcherds-Cartan super matrix

$$
A=\left(\begin{array}{ccc}
-k & -a & -b \\
-a & 2 & -1 \\
-b & -1 & 2
\end{array}\right)
$$

with charge $\underline{m}=\{r, 1,1\}$. Then for the root $\alpha=-p \alpha_{1}-q \alpha_{2}-t \alpha_{3} \in Q_{-}$, the dimension of $\mathfrak{g}_{\alpha}$ is

$$
\operatorname{Dim} \mathfrak{g}_{\alpha}=\sum_{\substack{s_{4}=0 \\\left(q-t+a s_{4}\right) \geq 0}}^{\min \left(p, q,\left[\frac{t}{a+1}\right]\right)} \frac{(p-1) !(-r)^{p}}{(p-q) !\left(q-t+a s_{4}\right) !\left(t-(a+1) s_{4}\right) ! s_{4} !} .
$$


Moreover the following combinatorial identity holds:

$$
\begin{gathered}
\sum_{\substack{s_{4}=0 \\
\left(q-t+a s_{4}\right) \geq 0}}^{\min \left(p, q,\left[\frac{t}{a+1}\right]\right)} \frac{(p-1) !(-r)^{p}}{(p-q) !\left(q-t+a s_{4}\right) !\left(t-(a+1) s_{4}\right) ! s_{4} !} \\
=\sum_{\phi \in T^{(J)}(\tau)} \frac{(p-1) !(-r)^{p}}{(p-q) !(q-|\phi|) ! \phi !} .
\end{gathered}
$$

REMARK 3.4. In the above formula, $(p-q) \geq 0$ and $\left(t-(a+1) s_{4}\right) \geq 0$ always hold. The term in the LHS containing $\left(q-t+a s_{4}\right)$ ! in the denominator, if $\left(q-t+a s_{4}\right)<0$, should be omitted.

EXAMPLE 3.5. For the Borcherds-Cartan super matrix

$$
A=\left(\begin{array}{ccc}
-k & -1 & -b \\
-1 & 2 & -1 \\
-b & -1 & 2
\end{array}\right)
$$

consider a root $\alpha=\tau=(5,3,4)$ with $\mathrm{r}=2$. Substituting $\alpha=\tau=(5,3,4), r=$ $2, a=1$ in (3.4), we have

$$
\begin{aligned}
W^{(J)}(\tau) & =\sum_{\substack{s_{4}=0 \\
\left(q-t+a s_{4}\right) \geq 0 \\
\min \left(5,3,\left[\frac{4}{2}\right]\right)}}^{\min \left(p, q,\left[\frac{t}{a+1}\right]\right)} \frac{(p-1) !(-r)^{p}}{(p-q) !\left(q-t+a s_{4}\right) !\left(t-(a+1) s_{4}\right) ! s_{4} !} \\
& =\sum_{s_{4}=1}^{2} \frac{(5-1) !(-2)^{5}}{(5-3) !\left(3-4+s_{4}\right) !\left(4-2 s_{4}\right) ! s_{4} !} \\
& =\sum_{s_{4}=1}^{2} \frac{4 !(-32)}{2 !\left(-1+s_{4}\right) !\left(4-2 s_{4}\right) ! s_{4} !} \\
& =\frac{4 !(-32)}{2 ! 0 ! 2 !}+\frac{4 !(-32)}{2 ! 1 ! 0 ! 2 !} \\
& =-192-192=-384 .
\end{aligned}
$$

Substituting $\alpha=\tau=(5,3,4), r=2, a=1$ in (3.5), we have

$$
\begin{aligned}
W^{(J)}(\tau) & =\sum_{\phi \in T^{(J)}(\alpha)} \frac{(p-1) !(-r)^{p}}{(p-q) !(q-|\phi|) ! \phi !} \\
& =\frac{4 !(-32)}{2 ! 2 ! 0 ! 1 !}+\frac{4(-32)}{2 ! 2 ! 0 ! 1 !}=-192-192=-384
\end{aligned}
$$

Hence the equality (3.6) holds. 
EXAMPLE 3.6. For the Borcherds-Cartan super matrix

$$
A=\left(\begin{array}{ccc}
-k & -2 & -b \\
-2 & 2 & -1 \\
-b & -1 & 2
\end{array}\right)
$$

consider a root $\alpha=\tau=(4,2,3) \in Q_{-}$with $\mathrm{r}=1$. Substituting $\alpha=\tau=$ $(4,2,3), r=1, a=2$ in (3.4), we have

$$
\begin{aligned}
W^{(J)}(\tau) & =\sum_{\substack{s_{4}=0 \\
\left(q-t+a s_{4}\right) \geq 0}}^{\min \left(p, q,\left[\frac{r}{a+1}\right]\right)} \frac{(p-1) !(-r)^{p}}{(p-q) !\left(q-t+a s_{4}\right) !\left(t-(a+1) s_{4}\right) ! s_{4} !} \\
& =\sum_{s_{4}=1}^{\min \left(4,2,\left[\frac{3}{3}\right]\right)} \frac{(4-1) !(-1)^{4}}{(4-2) !\left(2-3+2 s_{4}\right) !\left(3-3 s_{4}\right) ! s_{4} !} \\
& =\frac{3 !}{2 ! 1 ! 0 ! 1 !}=3 .
\end{aligned}
$$

Substituting $\alpha=\tau=(4,2,3), r=1, a=2$ in (3.5), we get

$$
\begin{aligned}
W^{(J)}(\tau) & =\sum_{\phi \in T^{(J)}(\tau)} \frac{(p-1) !(-r)^{p}}{(p-q) !(q-|\phi|) ! \phi !} \\
& =\frac{(4-1) !(-1)^{4}}{2 !(3-2) ! 1 ! 1 !}=3 .
\end{aligned}
$$

Hence the equality (3.6) holds.

3.3. Dimension Formula and combinatorial identity for the Borcherds superalgebra which is an extension of $A_{3}$. Here we are finding the superdimension formula and combinatorial identity for the Borcherds superalgebra which is an extension of $A_{3}$ using the same $J \subseteq I^{r e}$ and solving $T^{(J)}(\tau)$ in two different ways.

Consider the extended-hyperbolic Borcherds superalgebra $\mathfrak{g}=\mathfrak{g}(A, \underline{m}, C)$ associated with the extended-hyperbolic Borcherds-Cartan super matrix

$$
A=\left(\begin{array}{cccc}
-k & -a & -b & -c \\
-a & 2 & -1 & 0 \\
-b & -1 & 2 & -1 \\
-c & 0 & -1 & 2
\end{array}\right)
$$

and the corresponding coloring matrix be

$$
C=\left(\begin{array}{cccc}
-1 & c_{1} & c_{2} & c_{3} \\
c_{1}^{-1} & 1 & c_{4} & c_{5} \\
c_{2}^{-1} & c_{4}^{-1} & 1 & c_{6} \\
c_{3}^{-1} & c_{5}^{-1} & c_{6}^{-1} & 1
\end{array}\right)
$$


with $c_{1}, c_{2}, c_{3}, c_{4} \in C^{X}$. Let $I=\{1,2,3,4\}$ be the index set with charge $\underline{m}=\{r, 1,1,1\}$.

Let us consider the root $\alpha=k_{1} \alpha_{1}+k_{2} \alpha_{2}+k_{3} \alpha_{3}+k_{4} \alpha_{4} \in Q$. We have $\theta(\alpha, \alpha)=(-1)^{k_{1}^{2}}$. Hence $\alpha$ is an even root (resp. odd root) if $k_{1}$ is even integer (resp. odd integer). Also $T=\left\{\alpha_{1}\right\}$ (counted 'r' times) and the subset $F \subset T$ is either empty or $\left\{\alpha_{1}\right\}$. Take $J \subseteq I^{r e}$ as $J=\{2,3,4\}$. By Lemma 2.13, this implies that $W(J)=\{1\}$. Then

$$
\mathfrak{g}_{0}=\left\langle e_{2}, f_{2}, h_{2}, e_{3}, f_{3}, h_{3}, e_{4}, f_{4}, h_{4}\right\rangle \cong \operatorname{sl}(4, \mathbb{C}) .
$$

Using (2.7) and (2.8) we can write the homological spaces as

$$
\begin{aligned}
& H_{1}^{(J)}=V_{J}\left(1\left(\rho-\alpha_{1}\right)-\rho\right)=V_{J}\left(-\alpha_{1}\right), \\
& H_{k}^{(J)}=0 \quad \forall k \geq 2 .
\end{aligned}
$$

Therefore

$$
H^{(J)}=H_{1}^{(J)}=V_{J}\left(-\alpha_{1}\right) \oplus V_{J}\left(-\alpha_{1}\right) \oplus \ldots \oplus V_{J}\left(-\alpha_{1}\right) \text { (counted } r \text { times) }
$$

with

$$
\begin{aligned}
& \operatorname{Dim} H_{(1,0,0,0)}^{(J)}=-r, \quad \operatorname{Dim} H_{(1,1,0,0)}^{(J)}=-r, \quad \operatorname{Dim} H_{(1,1,1,0)}^{(J)}=-r, \\
& \operatorname{Dim} H_{(1,1,1,1)}^{(J)}=-r, \quad \operatorname{Dim} H_{(1,1,1, a+1)}^{(J)}=-r .
\end{aligned}
$$

Hence we have

$$
P\left(H^{(J)}\right)=\{(1,0,0,0),(1,1,0,0),(1,1,1,0),(1,1,1,1),(1,1,1, a+1)\} .
$$

Let $\alpha=\tau=-p \alpha_{1}-q \alpha_{2}-u \alpha_{3}-v \alpha_{4} \in Q_{-}$, with $(p, q, u, v) \in \mathbb{Z}_{\geq 0} \times$ $\mathbb{Z}_{\geq 0} \times \mathbb{Z}_{\geq 0} \times \mathbb{Z}_{\geq 0}$. Then by Proposition 2.17, we get

$$
\begin{aligned}
T^{(J)}(\tau)=\left\{\left(s_{1}, s_{2}, s_{3}, s_{4}, s_{5}\right) \mid\right. & s_{1}(1,0,0,0)+s_{2}(1,1,0,0)+s_{3}(1,1,1,0) \\
& \left.+s_{4}(1,1,1,1)+s_{5}(1,1,1, a+1)=(p, q, u, v)\right\} .
\end{aligned}
$$

This implies

$$
\begin{aligned}
s_{1}+s_{2}+s_{3}+s_{4}+s_{5} & =p, \\
s_{2}+s_{3}+s_{4}+s_{5} & =q, \\
s_{3}+s_{4}+s_{5} & =u, \\
s_{4}+(a+1) s_{5} & =v .
\end{aligned}
$$


We have

$$
\begin{aligned}
& s_{1}=p-q, \\
& s_{2}=q-u, \\
& s_{3}=u-v+s_{5}, \\
& s_{4}=v-(a+1) s_{5}, \\
& s_{5}=0 \text { to } \min \left(p, q, u,\left[\frac{v}{a+1}\right]\right) .
\end{aligned}
$$

Applying obtained formulae for $s_{1}, s_{2}, s_{3}, s_{4}, s_{5}$ in Witt partition formula (2.12) we get

$$
W^{(J)}(\tau)=\sum_{\substack{s_{5}=0 \\\left(u-v+s_{5}\right) \geq 0}}^{\min \left(p, q, u,\left[\frac{v}{a+1}\right]\right)} \frac{(p-1) !(-r)^{p}}{(p-q) !(q-r) !\left(u-v+s_{5}\right) !\left(v-(a+1) s_{5}\right) ! s_{5} !}
$$

(note that $(p-q) \geq 0,(q-r) \geq 0$ and $\left.\left(v-(a+1) s_{5}\right) \geq 0\right)$.

From (2.13), the dimension $\mathfrak{g}_{\alpha}$ is

$$
\begin{aligned}
\operatorname{Dim} \mathfrak{g}_{\alpha} & =\sum_{d \mid \alpha} \frac{1}{d} \mu(d) W^{(J)}\left(\frac{\alpha}{d}\right) \\
& =\sum_{d \mid \alpha} \frac{1}{d} \mu(d) \sum_{n \in T^{(J)}\left(\frac{\alpha}{d}\right)} \frac{(|n|-1) !}{n !} \prod D(i)^{n_{i}} .
\end{aligned}
$$

Substituting the value of $W^{(J)}(\tau)$ from (3.7) in the above dimension formula yields

$$
\operatorname{Dim} \mathfrak{g}_{\alpha}=\sum_{\substack{s_{5}=0 \\\left(u-v+s_{5}\right) \geq 0}}^{\min \left(p, q, u,\left[\frac{v}{a+1}\right]\right)} \frac{(p-1) !(-r)^{p}}{(p-q) !(q-r) !\left(u-v+s_{5}\right) !\left(v-(a+1) s_{5}\right) ! s_{5} !}
$$

(note that $(p-q) \geq 0,(q-r) \geq 0$ and $\left(v-(a+1) s_{5}\right) \geq 0$ ).

If we solve the same $P\left(H^{(J)}\right)$ using partition and substituting the partition, we obtain

$$
T^{(J)}(\tau)=\{(p-q), q-u, u-|\phi|, \phi\},
$$

where $\phi$ is partition of $v$ with parts $\leq(1, a+1)$ and of length $v$. Applying $T^{(J)}(\tau)$ in Witt partition formula $(2.12)$

$$
W^{(J)}(\tau)=\sum_{\phi \in T^{(J)}(\tau)} \frac{(p-1) !(-r)^{p}}{(p-q) !(q-u) !(u-|\phi|) ! \phi !} .
$$


From equation (2.13), the dimension $\mathfrak{g}_{\alpha}$ is

$$
\begin{aligned}
\operatorname{Dim} \mathfrak{g}_{\alpha} & =\sum_{d \mid \alpha} \frac{1}{d} \mu(d) W^{(J)}\left(\frac{\alpha}{d}\right) \\
& =\sum_{d \mid \alpha} \frac{1}{d} \mu(d) \sum_{n \in T^{(J)}\left(\frac{\alpha}{d}\right)} \frac{(|n|-1) !}{n !} \prod D(i)^{n_{i}} .
\end{aligned}
$$

Substituting the value of $W^{(J)}(\tau)$ from (3.8) in the above dimension formula, we obtain

$$
\operatorname{Dim} \mathfrak{g}_{\alpha}=\sum_{\phi \in T^{(J)}(\tau)} \frac{(p-1) !(-r)^{p}}{(p-q) !(q-u) !(u-|\phi|) ! \phi !} .
$$

Consider the equation (3.8),

$$
W^{(J)}(\alpha)=\sum_{\phi \in T^{(J)}(\tau)} \frac{(p-1) !(-r)^{p}}{(p-q) !(q-u) !(u-|\phi|) ! \phi !}
$$

where $\phi$ is partitions of $v$ in $\alpha=\tau=-p \alpha_{1}-q \alpha_{2}-u \alpha_{3}-v \alpha_{4}$ with parts $(1, \mathrm{a}+1)$ of length $\leq v$. That is, $\phi$ can be written as $\left(n_{1}, n_{(a+1)}\right)$, where $n_{1}$ is number of 1's in the partition of $v$ and $n_{(a+1)}$ is number of $(a+1)$ 's in the same partition of $v$. Then $\phi=\{(v, 0),(v-(a+1), 1),(v-2(a+$ $1), 2), \ldots$ up to the term satisfying $\left.v-n_{1}(a+1)>0\right\}$.

We have

$$
\begin{aligned}
W^{(J)}(\tau)= & \sum_{n_{1}, n_{(a+1)} \in T^{(J)}(\tau)} \frac{(p-1) !(-r)^{p}}{(p-q) !(q-u) !\left(u-n_{1}-n_{(a+1)}\right) ! n_{1} ! n_{(a+1)} !} \\
= & \frac{(p-1) !(-r)^{p}}{(p-q) !(q-u) !(u-v-0) ! t ! 0 !} \\
& +\frac{(p-1) !(-r)^{p}}{(p-q) !(q-u) !(u-v+a) !(v-(a+1)) ! 1 !} \\
& +\frac{(p-1) !(-r)^{p}}{(p-q) !(q-u) !(u-v+2 a) !(v-2(a+1)) ! 2 !} \\
& +\cdots \text { up to the term satisfying } v-n_{1}(a+1)>0 .
\end{aligned}
$$

Here we are considering the roots of type $(p, q, u, v)$ such that $p \geq q, u \leq v$ and also $p \geq v$. Moreover the sum ranges up to the term satisfying $v-n_{1}(a+1)>0$. So we can write the above summation as

$$
\operatorname{Dim} \mathfrak{g}_{\alpha}=\sum_{\substack{s_{5}=0 \\\left(u-v+s_{5}\right) \geq 0}}^{\min \left(p, q, u,\left[\frac{v}{a+1}\right]\right)} \frac{(p-1) !(-r)^{p}}{(p-q) !(q-r) !\left(u-v+s_{5}\right) !\left(v-(a+1) s_{5}\right) ! s_{5} !}
$$

which is same as equation (3.7) (note that $(p-q) \geq 0,(q-r) \geq 0$ and $\left.\left(v-(a+1) s_{5}\right) \geq 0\right)$. 
Hence we proved the following theorem:

TheOREM 3.7. Let $\mathfrak{g}=\mathfrak{g}(A, \underline{m}, C)$ be the extended-hyperbolic Borcherds superalgebra associated with the extended-hyperbolic Borcherds-Cartan super matrix

$$
A=\left(\begin{array}{cccc}
-k & -a & -b & -c \\
-a & 2 & -1 & 0 \\
-b & -1 & 2 & -1 \\
-c & 0 & -1 & 2
\end{array}\right)
$$

with charge $\underline{m}=\{r, 1,1,1\}$. Then for the root $\alpha=\tau=-p \alpha_{1}-q \alpha_{2}-u \alpha_{3}-$ $v \alpha_{4} \in Q_{-}$, the dimension of $\mathfrak{g}_{\alpha}$ is

$$
\operatorname{Dim} \mathfrak{g}_{\alpha}=\sum_{\substack{s_{5}=0 \\\left(u-v+s_{5}\right) \geq 0}}^{\min \left(p, q, u,\left[\frac{v}{a+1}\right]\right)} \frac{(p-1) !(-r)^{p}}{(p-q) !(q-r) !\left(u-v+s_{5}\right) !\left(v-(a+1) s_{5}\right) ! s_{5} !}
$$

Moreover the following combinatorial identity holds:

$$
\begin{gathered}
\sum_{\substack{s_{5}=0 \\
\left(u-v+s_{5}\right) \geq 0}}^{\min \left(p, q, u,\left[\frac{v}{a+1}\right]\right)} \frac{(p-1) !(-r)^{p}}{(p-q) !(q-u) !\left(u-v+s_{5}\right) !\left(v-(a+1) s_{5}\right) ! s_{5} !} \\
=\sum_{\phi \in T^{(J)}(\tau)} \frac{(p-1) !(-r)^{p}}{(p-q) !(q-u) !(u-|\phi|) ! \phi !} .
\end{gathered}
$$

REMARK 3.8. In the above formula, $(p-q) \geq 0,(q-r) \geq 0$ and $(v-(a+$ 1) $\left.s_{5}\right) \geq 0$ always hold. The term in the LHS containing $\left(u-v+s_{5}\right)$ ! in the denominator, if $\left(u-v+s_{5}\right)<0$ should be omitted.

EXAMPLE 3.9. For the Borcherds-Cartan super matrix

$$
A=\left(\begin{array}{cccc}
-k & -1 & -b & -c \\
-1 & 2 & -1 & 0 \\
-b & -1 & 2 & -1 \\
-c & 0 & -1 & 2
\end{array}\right)
$$


consider the root $\alpha=\tau=(5,3,2,4)$ with $r=2$ and $a=1$. Using these values in $(3.7)$, we have

$$
\begin{aligned}
\sum_{\substack{s_{5}=0 \\
\left(u-v+s_{5}\right) \geq 0}}^{\min \left(p, q, u,\left[\frac{v}{a+1}\right]\right)} \frac{(p-1) !(-r)^{p}}{(p-q) !(q-u) !\left(u-v+s_{5}\right) !\left(v-(a+1) s_{5}\right) ! s_{5} !} \\
=\sum_{s_{5}=2}^{\min \left(5,3,2,\left[\frac{4}{2}\right]\right)} \frac{(5-1) !(-2)^{5}}{(5-3) !(3-2) !(2-4+2) !(4-2(2)) ! 2 !} \\
=\frac{4 !(-2)^{5}}{2 ! 1 ! 0 ! 0 ! 2 !} \\
=-192 .
\end{aligned}
$$

Now if we use $\alpha=(p, q, u, v)=(5,3,2,4), r=2, a=1$ in (3.8), we have

$$
\sum_{\phi \in T^{(J)}(\tau)} \frac{(p-1) !(-r)^{p}}{(p-q) !(q-u) !(u-|\phi|) ! \phi !}=\frac{(5-1) !(-2)^{5}}{(5-3) !(3-2) !(2-|\phi|) ! \phi !}=-192 .
$$

Hence the equality (3.9) holds.

EXAMPLE 3.10. For the Borcherds-Cartan super matrix

$$
A=\left(\begin{array}{cccc}
-k & -1 & -b & -c \\
-1 & 2 & -1 & 0 \\
-b & -1 & 2 & -1 \\
-c & 0 & -1 & 2
\end{array}\right)
$$

consider the root $\alpha=\tau=(7,4,3,5)=(p, q, u, v)$ with $\mathrm{r}=1$ and $\mathrm{a}=1$. Applying in equation (3.7), we have

$$
\begin{aligned}
\sum_{\substack{s_{5}=0 \\
\left(u-v+s_{5}\right) \geq 0}}^{\min \left(p, q, u,\left[\frac{v}{a+1}\right]\right)} \frac{(p-1) !(-r)^{p}}{(p-q) !(q-u) !\left(u-v+s_{5}\right) !\left(v-(a+1) s_{5}\right) ! s_{5} !} \\
=\sum_{s_{5}=1}^{2} \frac{(7-1) !(-1)^{7}}{(7-4) !(4-3) !\left(3-5+2 s_{5}\right) !\left(3-2 s_{5}\right) ! s_{5} !} \\
=\frac{6 !(-1)}{3 ! 0 ! 1 ! 1 !}+\frac{6 !(-1)}{3 ! 1 ! 2 !(-1) ! 2 !} \\
=-120
\end{aligned}
$$

Consider the $\operatorname{root} \tau=\alpha$ as $(7,4,3,5)$ with $\mathrm{r}=1$ and $\mathrm{a}=1$. Applying in equation (3.8), we have

$$
\sum_{\phi \in T^{(J)}(\tau)} \frac{(p-1) !(-r)^{p}}{(p-q) !(q-u) !(u-|\phi|) ! \phi !}=\frac{(7-1) !(-1)^{7}}{(7-4) !(4-3) !(3-2) ! 1 !}=-120 .
$$

Hence the equality (3.9) holds. 
REMARK 3.11. In the above two Sections 3.2 and 3.3., the identities (3.6) and (3.9) hold for any root, because we have derived the identities by simply solving the $T^{(J)}(\tau)$ in two different ways.

3.4. Superdimension Formula for a extended-hyperbolic Borcherds superalgebra which is an extension of hyperbolic Kac-Moody algebra. Now, we find superdimension formula extended-hyperbolic Borcherds superalgebra which is an extension of hyperbolic Kac-Moody algebra.

Proposition 3.12. Let $\mathfrak{g}=\mathfrak{g}(A, \underline{m}, C)$ be the extended-hyperbolic Borcherds superalgebra associated with the extended-hyperbolic BorcherdsCartan super matrix

$$
A=\left(\begin{array}{ccc}
-2 & -1 & -1 \\
-2 & 2 & -4 \\
-1 & -2 & 2
\end{array}\right)
$$

and $I=\{1,2,3\}$ be the index set with charge $\underline{m}=\{1,1,1\}$. Then for the root $\alpha=-l \alpha_{1}-m \alpha_{2}-n \alpha_{3} \in Q^{-}$with $l, m, n \in \mathbb{Z}_{\geq 0}$ and weight $\lambda=-\alpha_{1}-m \alpha_{2}-$ $n \alpha_{3}$, prove that $\operatorname{Dim} \mathfrak{g}_{\alpha}=\theta(\lambda, \lambda) p\left(m-(m-n)^{2}\right)$.

Proof. Consider the extended-hyperbolic Borcherds superalgebra $\mathfrak{g}=$ $\mathfrak{g}(A, \underline{m}, C)$ associated with the extended-hyperbolic Borcherds-Cartan super matrix

$$
A=\left(\begin{array}{ccc}
-2 & -1 & -1 \\
-2 & 2 & -4 \\
-1 & -2 & 2
\end{array}\right)
$$

and the corresponding coloring matrix be

$$
C=\left(\begin{array}{ccc}
-1 & c_{1} & c_{2} \\
c_{1}^{-1} & 1 & c_{3}^{(-1)} \\
c_{2} & c_{3} & 1
\end{array}\right)
$$

with $c_{1}, c_{2}, c_{3} \in C^{X}$.

Let us consider the root $\alpha=k_{1} \alpha_{1}+k_{2} \alpha_{2}+k_{3} \alpha_{3} \in Q$. We have $\theta(\alpha, \alpha)=$ $(-1)^{k_{1}^{2}+k_{2}^{2}}$. Also $T=\left\{\alpha_{1}\right\}$ and the subset $F \subset T$ is either empty or $\left\{\alpha_{1}\right\}$. Take $J \subset I^{r e}$ as $J=\{2,3\}$. By Lemma 2.13, this implies that $W(J)=\{1\}$. From (2.7) and (2.8), we have

Therefore

$$
\begin{aligned}
& H_{1}^{(J)}=V_{J}\left(1\left(\rho-\alpha_{1}\right)-\rho\right)=V_{J}\left(-\alpha_{1}\right), \\
& H_{k}^{(J)}=0 \text { for all } k \geq 2 .
\end{aligned}
$$

$$
H^{(J)}=H_{1}^{(J)}=V_{J}\left(-\alpha_{1}\right) .
$$

Let $\alpha=-l \alpha_{1}-m \alpha_{2}-n \alpha_{3} \in Q^{-}$such that $l, m, n \in \mathbb{Z}_{\geq 0}$. Then from Frenkel and Kac (1980), we have weights of $V_{J}\left(-\alpha_{1}\right)$ is of the form $(1, m, n)$ where $m$ and $n$ are non-negative integers satisfying the inequality $m-(m-n)^{2} \geq 0$.

So, for $m=0,1,2, \ldots, n$ ranges from $m-\sqrt{m}$ to $m+\sqrt{m}$. 
For a weight $\lambda=(1, m, n)$ by Kang ([11]), we get

$$
\operatorname{dim}\left(V_{J}\left(-\alpha_{1}\right)\right)_{\lambda}=p\left(m-(m-n)^{2}\right) .
$$

Using

we get

$$
-\frac{(\lambda, \lambda)}{2}=m-(m-n)^{2}
$$

$$
\operatorname{dim}\left(V_{J}\left(-\alpha_{1}\right)\right)_{\lambda}=p\left(-\frac{(\lambda, \lambda)}{2}\right)
$$

where $p(n)$ is

$$
\sum_{n=0}^{\infty} p(n) q^{n}=\frac{1}{\phi(q)}=\frac{1}{\prod_{j \geq 1}\left(1-q^{j}\right)}
$$

Then

$$
\operatorname{Dim}\left(V_{J}\left(-\alpha_{1}\right)\right)_{\lambda}=\theta(\lambda, \lambda) p\left(m-(m-n)^{2}\right) .
$$

Here we notice that every root $\alpha$ of $\mathfrak{g}$ is of the form $\alpha=(l, m, n)$ with $l \geq 1$ and $m, n \geq 0$. We have

$$
T^{(J)}(\alpha)=\left\{n=\left(n_{i}\right)_{i \geq 0} \mid \sum n_{i} \alpha_{i}=\alpha\right\} .
$$

Then for a root $\alpha=-l \alpha_{1}-m \alpha_{2}-n \alpha_{3} \in Q_{-}$with $l \geq 1$ and $m, n \geq 0$ the superdimension is

$$
\begin{aligned}
\operatorname{Dim} \mathfrak{g}_{\alpha} & =\sum_{d \mid \alpha} \frac{\mu(d)}{d} \sum_{n \in T^{J}(\alpha)} \frac{(|n|-1) !}{n !} \prod_{i}\left(\operatorname{Dim} H_{\alpha_{i}}^{J}\right)^{n_{i}}, \\
\operatorname{Dim} \mathfrak{g}_{\alpha} & =\theta(\lambda, \lambda) p\left(m-(m-n)^{2}\right) .
\end{aligned}
$$

REMARK 3.13. In Section 3.4, for the above Borcherds superalgebra which is an extension of hyperbolic Kac-Moody algebra, the Weyl group is infinite. So, we are using the results from Kang ([11]) to find the superdimension formula.

EXAMPLE 3.14. Consider the extended-hyperbolic Borcherds superalgebra $\mathfrak{g}=\mathfrak{g}(A, \underline{m}, C)$ associated with the extended-hyperbolic Borcherds-Cartan super matrix

$$
A=\left(\begin{array}{ccc}
-2 & -1 & -1 \\
-2 & 2 & -4 \\
-1 & -2 & 2
\end{array}\right)
$$

and the corresponding coloring matrix be

$$
C=\left(\begin{array}{ccc}
-1 & c_{1} & c_{2} \\
c_{1}^{-1} & 1 & c_{3} \\
c_{2}^{-1} & c_{3}^{-1} & 1
\end{array}\right)
$$

with $c_{1}, c_{2}, c_{3} \in C^{X}$. 
Let $I=\{1,2,3\}$ be the index set with charge $\underline{m}=\{1,1,1\}$.

Then for a root $\alpha=-l \alpha_{1}-m \alpha_{2}-n \alpha_{3} \in Q_{-}$with $l \geq 1$ and $m, n \geq 0$ the superdimension is

$$
\begin{aligned}
\operatorname{Dim} \mathfrak{g}_{\alpha} & =\sum_{d \mid \alpha} \frac{\mu(d)}{d} \sum_{n \in T^{J}(\alpha)} \frac{(|n|-1) !}{n !} \prod_{i}\left(\operatorname{Dim} H_{\alpha_{i}}^{J}\right)^{n_{i}}, \\
\operatorname{Dim} g_{\alpha} & =\theta(\lambda, \lambda) p\left(m-(m-n)^{2}\right) .
\end{aligned}
$$

TABLE 1. The following table gives some of the weights and their dimensions

\begin{tabular}{cccc}
\hline Weights & Dimensions & Weights & Dimensions \\
\hline$(1,0,0)$ & -1 & $(1,1,0)$ & 1 \\
$(1,2,1)$ & -1 & $(1,1,1)$ & 1 \\
$(1,2,2)$ & -2 & $(1,3,2)$ & 2 \\
$(1,4,3)$ & -3 & $(1,3,3)$ & 3 \\
$(1,4,2)$ & -1 & $(1,3,4)$ & 2 \\
$(1,4,4)$ & -5 & $(1,5,4)$ & 5 \\
$(1,4,5)$ & -3 & $(1,5,3)$ & 1 \\
$(1,4,3)$ & -3 & $(1,5,5)$ & 6
\end{tabular}

For the root $(2,4,5)$, applying the above Proposition 3.12, the Dimension of $\mathfrak{g}$ at $(2,4,5)$ is equal to $(-1)(-3)+(1)(2)+(-2)(-1)+(3)(1)=10$.

REMARK 3.15. We hope that, in general, superdimensions of roots and the corresponding combinatorial identities for Borcherds Superalgebras which are extensions of all finite dimensional Kac-Moody algebras and superdimensions for all other categories can also be found out.

\section{ACKNOWLEDGEMENTs.}

The research has been financially supported by the University Grants Commission (UGC), Govt. of India, through the Major research Project, F.No.36-272/2008(SR). The authors, N. Sthanumoorthy (Principal Investigator of the Project) and K. Priyadharsini (Project Fellow for the Project) are very much thankful to the UGC for the same.

The authors are also thankful to the referee for pointing out a few misprints in the initial version of the paper.

\section{REFERENCES}

[1] R. E. Borcherds, Generalized Kac-Moody algebras, J. Algebra 115 (1988), 501-512.

[2] R. E. Borcherds, Monstrous moonshine and monstrous Lie superalgebras, Invent. Math. 109 (1992), 405-444.

[3] A. J. Feingold and I. B. Frenkel, A hyperbolic Kac-Moody algebra and the theory of Siegel modular forms of genus 2, Math. Ann. 263 (1983), 87-144. 
[4] I. B. Frenkel and V. G. Kac, Basic representation of affine Lie algebras and dual resonance models, Invent. Math. 62 (1980), 23-66.

[5] J. Hontz and K.C. Misra, Root multiplicities of the indefinite Kac-Moody Lie algebras $H D_{4}^{(3)}$ and $H G_{2}^{(1)}$, Comm. Algebra 30 (2002), 2941-2959.

[6] V. G. Kac, Lie superalgebras, Advances in Math. 26 (1977), 8-96.

[7] V. G. Kac, Infinite dimensional algebras, Dedekind's $\eta$ - function, classical Mobius function and the very strange formula, Adv. in Math. 30 (1978), 85-136.

[8] V.G. Kac, Infinite dimensional Lie algebras, 3rd ed. Cambridge University Press., Cambridge, 1990.

[9] V. G. Kac and M. Wakimoto, Integrable highest weight modules over affine superalgebras and number theory, in: Lie theory and geometry, Birkhäuser Boston, Boston, 1994, 415-456.

[10] S.-J. Kang, Kac-Moody Lie algebras, spectral sequences, and the Witt formula, Trans. Amer. Math. Soc. 339 (1993), 463-493.

[11] S. J. Kang, Root multiplicities of the hyperbolic Kac-Moody Lie algebra $H A_{1}^{(1)}$, J. Algebra 160 (1993), 492-523.

[12] S. J. Kang, Generalized Kac-Moody algebras and the modular function j, Math. Ann. 298 (1994), 373-384.

[13] S. J. Kang, Root multiplicities of Kac-Moody algebras, Duke Math. J. 74 (1994), 635-666.

[14] S. J. Kang, On the hyperbolic Kac-Moody Lie algebra $H A_{1}^{(1)}$, Trans. Amer. Math. Soc. 341 (1994), 623-638.

[15] S. J. Kang, Root multiplicities of graded Lie algebras, in Lie algebras and Their Representations, S. J. Kang, M. H. Kim, I. S. Lee (eds), Amer. Math. Soc., Providence, 1996, 161-176.

[16] S. J. Kang, Graded Lie superalgebras and the superdimension formula, J. Algebra 204 (1998), 597-655.

[17] S. J. Kang and M. H. Kim, Borcherds superalgebras and a monstrous Lie superalgebras, Math. Ann. 307 (1997), 677-694.

[18] S. J. Kang and M. H. Kim, Dimension formula for graded Lie algebras and its applications, Trans. Amer. Math. Soc. 351 (1999), 4281-4336.

[19] S. J. Kang and D. J. Melville, Root multiplicities of the Kac-Moody algebras $H A_{n}^{(1)}$, J. Algebra 170 (1994), 277-299.

[20] S. Kass, R. V. Moody, J. Patera and R. Slansky, Affine Lie algebras, weight multiplicities and branching rules. 1, University of California Press, Berkeley, 1990.

[21] K. Kim and D. U. Shin, The recursive dimension formula for graded Lie algebras and its applications, Comm. Algebra 27 (1999), 2627-2652.

[22] L. S. Liu,Kostant's formula for Kac-Moody Lie superalgebras, J. Algebra 149 (1992), $155-178$.

[23] M. Miyamoto, A generalization of Borcherds algebra and denominator formula, J. Algebra 180 (1996), 631-651.

[24] M. Scheunert, The theory of Lie superalgebras. An introduction, Springer, Berlin, 1979 .

[25] N. Sthanumoorthy and A. Uma Maheswari, Purely imaginary roots of Kac-Moody algebras, Comm. Algebra 24 (1996), 677-693.

[26] N. Sthanumoorthy and A. Uma Maheswari, Root multiplicities of extended-hyperbolic Kac-Moody algebras, Comm. Algebra 24 (1996), 4495-4512.

[27] N. Sthanumoorthy and P. L. Lilly, On the root systems of generalized Kac-Moody algebras, The Journal of Madras University, Section B Sciences 52 (2000), 81-102.

[28] N. Sthanumoorthy and P. L. Lilly, Special imaginary roots of generalized Kac-Moody algebras, Comm. Algebra 10 (2002), 4771-4787. 
[29] N. Sthanumoorthy and P. L. Lilly, A note on purely imaginary roots of generalized Kac-Moody algebras, Comm. Algebra 31 (2003), 5467-5479.

[30] N. Sthanumoorthy and P. L. Lilly, On some classes of root systems of generalized Kac-Moody algebras, in Kac-Moody Lie algebras and related topics, 2004, 289-313.

[31] N. Sthanumoorthy, P. L. Lilly and A. Uma Maheswari, Root multiplicities of some classes of extended hyperbolic Kac-Moody and extended hyperbolic generalized KacMoody algebras, in Kac-Moody Lie algebras and related topics, 2004, 315-347.

[32] N. Sthanumoorthy, P. L. Lilly and A. Uma Maheswari, Extended hyperbolic KacMoody algebras $\mathrm{EH}_{2}^{(2)}$ structure and root multiplicities, Comm. Algebra 32 (2004), 2457-2476.

[33] N. Sthanumoorthy and P. L. Lilly, Complete classifications of generalized Kac-Moody algebras possessing special imaginary roots and strictly imaginary property, Comm. Algebra 35 (2007), 2450-2471.

[34] N. Sthanumoorthy and P. L. Lilly, Root multiplicities of some generalized Kac-Moody algebras, Indian J. Pure Appl. Math. 38 (2007), 55-78.

[35] N. Sthanumoorthy, P. L. Lilly and A. Nazeer Basha, Special imaginary roots of BKM Lie super algebras, Int. J. Pure Appl. Math. 38 (2007), 513-542.

[36] N. Sthanumoorthy, P. L. Lilly and A. Nazeer Basha, Root supermultiplicities of some Borcherds superalgebras, Comm. Algebra 37 (2009), 1353-1388.

[37] N. Sthanumoorthy, P. L. Lilly and A. Nazeer Basha, Strictly imaginary roots and purely imaginary roots of BKM Lie superalgebras, Comm. Algebra 37 (2009), 23572390 .

[38] N. Sthanumoorthy and K. Priyadharsini, Complete classification of BKM Lie superalgebras possessing special imaginary roots, Applied Mathematics 2(4) (2012), 100-115.

[39] N. Sthanumoorthy and K. Priyadharsini, Complete classification of BKM Lie superalgebras possessing special imaginary roots, Comm. Algebra 41 (2013), 367400 .

N. Sthanumoorthy

The Ramanujan Institute for Advanced study in Mathematics University of Madras

Chennai - 600005

India

E-mail: sthanun@yahoo.com

K. Priyadharsini

The Ramanujan Institute for Advanced study in Mathematics

University of Madras

Chennai - 600005

India

E-mail: priyariasm@gmail.com

Received: 15.2.2013.

Revised: 5.9.2013. 